\title{
Earnings Management and Measurement Error
}

Christian Hofmann, Department of Business Administration and Managerial Accounting, University of Mannheim, Germany, E-Mail: christian.hofmann@bwl.uni-mannheim.de

\begin{abstract}
In this paper I consider the impact of a noisy indicator regarding a manager's manipulative behavior on optimal effort incentives and the extent of earmings management. The analysis in this paper extends a twotask, single performance measure LEN model by including a binary random variable. I show that contracting on the noisy indicator variable is not always useful. More specifically, the principal uses the indicator variable to prevent earnings management only under conditions where manipulative behavior is not excessive. Thus, under conditions of excessive earnings management, accounting adjustments that yield a more congruent overall performance measure can be more effective than an appraisal of the existence of earnings management to mitigate the earnings management problem.
\end{abstract}

Keywords: Earmings Management, Incentives, Indicator Variable, LEN Model, Performance Measurement.

Manuscript received June 3, 2008, accepted by Rainer Niemann (Accounting) September 19, 2008.

\section{INTRODUCTION}

Empirical evidence indicates that earnings management is widespread in practice. ${ }^{1}$ For example, managers use accounting judgment and real transactions to influence the expectations of capital market participants and to increase their earningsbased bonus awards.2,3 Frequently, earnings manipulation or window dressing is the consequence of incentives based on poor performance evaluation (Merchant 1980), i.e., the manager's performance measure does not correctly reflect firm value. Then, conceptually, there are two ways to remedy the earnings management problem:

a) directly, by performing accounting adjustments that yield a more congruent overall performance measure;

\footnotetext{
${ }^{1}$ Lev (2003) offers some illustrative examples.

2 Healy and Whalen (1999) discuss different incentives for earnings management.

3 See Healy (1985); Gaver, Gaver, and Austin (1995); Holthausen, Larcker, and Sloan (1995); Klein (2002); and Leuz, Nanda, and Wysocki (2003) for evidence of earnings management at the firmlevel, and Guidry, Leone, and Rock (1999) with respect to evidence at the business-unit level.
}

b) indirectly, by collecting and evaluating evidence to appraise whether or not the manager used earnings management to inflate reported earnings.

Essentially, (a) requires the release of a report on the magnitude of earnings management, whereas (b) comprises the release of a zero/one-signal that indicates the existence of earnings management. This paper's objective is to analyze the effectiveness of both approaches to addressing the earnings management problem.

For convenience, a report on the magnitude and the existence of earnings management can be related to audits performed by external and internal auditors. 4 While an external auditor expresses his opinion on whether a firm's financial statement is free of material misstatements and quantifies the influence of earnings management on the firm's financial posi-

4 Section 2.2 comprises a more technical description of the underlying assumptions regarding the auditing processes. 
tion $^{5}$ (i.e., he issues a report on the magnitude of earnings management), an internal auditor appraises the control framework and governance of the firm, and reports on the compliance with firm policies (comprising, e.g., a report on the existence of earnings management).

Either report is especially of relevance in situations characterized by a short-term relation between the manager who exerts the manipulative effort and his superior, e.g., because the manager expects to be promoted to a new job. Then, any reversals that are inherent in earnings management do not affect the manager's future compensation, such that earnings manipulation can result in a substantial wealth transfer to the manager. A similar argument applies to settings where institutional constraints impose a finite (i.e., short-term) contract, and where contracts are subject to mutually beneficial renegotiation (Hofmann 2004). In summary, the subsequent analysis investigates a setting where a noncongruent performance measure motivates legal earnings management and unfeasible long-term contracts prevent the inherent reversion of earnings management to be instrumental in ex-post settling up.

While common intuition suggests that by attaching a sufficiently high penalty to the indicator variable on the existence of earnings management, the principal is able to preclude earnings management, the consequences for effort incentives and regarding the relative effectiveness of the two approaches are less clear. In summary, I find, firstly, that preventing earnings management by use of an indicator variable does not, in general, coincide with stronger effort incentives. Secondly, (partly) filtering earnings manipulation to achieve a more congruent overall performance measure can actually increase the agent's effort to manipulate earnings. Nevertheless, under conditions that yield excessive earnings management, these accounting adjustments can be more effective in reducing earnings management than internal audits that provide a yes/no-statement regarding the manager's attempts to manipulate the books.

Key to my results is that both approaches to addressing the earnings management problem yield

\footnotetext{
5 For example, in Germany, § 321 Abs. 2 Satz 4 HGB in conjunction with IDW PS 450 (74) specify that the auditor has to quantify the impact of earnings management on the firm's net assets, financial position, and results of operations. Similar rules apply, e.g., to the UK via U10 ICAEW.
}

noisy reports. For example, following Watts and Zimmerman (1978), external auditors typically differ with respect to the cost of measuring earnings management, suggesting that an external auditor will provide a noisy measure of the extent to which reported earnings deviate from true earnings. Similarly, the process of collecting and evaluating evidence by the internal auditor regarding the existence of earnings management is, most likely, noisy as well (Ijiri and Kaplan 1971). Moreover, ignoring fraudulent accounting practices in the subsequent analysis increases the noisiness of both auditors' reports, since it is more difficult to distinguish earnings management from the legitimate exercise of accounting discretion for those judgments and estimates that fall within GAAP (Dechow and Skinner 2000).

Basically, the measurement error inherent in the indicator is twofold: A Type I error (false positive) arises if the indicator signals earnings management, although the manager did not exert manipulative effort. On the other hand, a Type II error (false negative) exists if the indicator signals no earnings management, while the manager did exert manipulative effort. The magnitude of both errors depends on the internal auditor's diligence in collecting and evaluating evidence and the Type I error will, in general, differ from the Type II error. Moreover, I assume that even if the indicator variable signals the existence of earnings management, its impact on reported earnings cannot be "undone". Hence, the focus of the internal audit is on detecting the compliance with firm policies regarding overstatements or understatements in financial reports, rather than releasing a report on "true earnings".

In the analysis, I consider a setting where an agent exerts productive and manipulative effort, an aggregate performance measure reports on both actions, an external auditor's report signals the magnitude of earnings management, and an internal auditor's report signals whether the manager performed earnings management. To simplify the analysis, I assume that the compensation contract is restricted to be a linear function of the contractible performance measures. Thus, the analysis extends the multi-task LEN model by including a supplemental binary random variable in the contract offered to the agent. At least two reasons motivate the use of the LEN model: First, linear contracts are frequently observed in practice. Secondly, the tractability in- 
herent in the LEN model offers a clear advantage compared with the analysis of optimal contracts.

Section 3 considers the impact of a report on the magnitude of earnings management. Interestingly, I find that while the external auditor's report can improve the congruency of the overall performance measure, including this report may actually increase the agent's effort to manipulate earnings. Key to this result is that the weight chosen for the external report optimizes the congruence of the overall measure plus the incentive risk imposed on the agent, and that the second objective can dominate the first. Then, by including the external report in the agent's performance evaluation the principal selects stronger effort incentives, and the stronger incentives more than make up for the reduced sensitivity of the overall measure to window dressing.

In Section 4, I consider the report on the existence of earnings management. Intuitively, the penalty necessary to keep the agent from manipulating earnings increases in effort incentives (Section 4.2). Also, precluding earnings management via a penalty in the case of an earnings management signal is costly to the principal, since she needs to compensate the agent for the additional risk inherent in this noisy signal. Thus, in Section 4.3 I identify conditions under which the principal does not use the indicator variable although it is informative about the agent's manipulative effort. In particular, the principal precludes earnings management by use of the indicator variable if the indicator is sufficiently informative (i.e., for relatively low Type I and Type II errors) and if the agent is not too productive. While this result corresponds, in general, to the informativeness principle (Holmstrom 1979), its consequence is that earnings management is either suppressed or not affected at all by including the indicator variable in the agent's contract.

Moreover, while contracting on the indicator variable keeps the agent from exerting manipulative effort, I find that conditions exist under which the principal optimally selects weaker effort incentives as compared to the setting without the indicator variable (Section 4.4). The key to this result is that the penalty necessary to suppress earnings management increases with effort incentives, and that the risk premium for a noisy indicator variable increases with the size for the penalty. Hence, one cannot state that suppressing earnings management by means of, e.g., evidence on the agent's manipu- lative behavior, always results in stronger incentives.

Finally, my analysis shows that the direct and indirect approach to addressing the earnings management problem have starkly different consequences for the agent's earnings management behavior. While including a report on the magnitude of earnings management in the agent's contract may actually increase the level of earnings management, including a noisy indicator variable weakly decreases the agent's effort to manipulate earnings. However, the noisy indicator will be included only for conditions where the extent of manipulative effort is rather low. In particular, under conditions of extensive earnings management, the availability of a noisy indicator variable does not help the principal in precluding earnings management. Then, a combination of the two approaches can be beneficial to the principal: given that accounting adjustments sufficiently reduce the (intended) level of earnings management, the noisy indicator variable can be effective in eliminating earnings management.

The paper is related to at least two lines of research: The first line considers the consequences of earnings management on effort incentives. Feltham and Xie (1994) demonstrate that window dressing results in weaker effort incentives. Moreover, Smith (2002) considers the impact of improving shortterm performance at the expense of a non-financial, long-term measure. Whereas, in both papers, earnings management is precluded only under knifeedge conditions, I show that with an indicator variable it is optimal for the principal to suppress earnings management for a wider range of parameter values. On the other hand, several papers address the question regarding under which conditions earnings management is in the best interest of the shareholders. Arya, Glover, and Sunder (1998) and Hofmann (2004) show that enabling self-interested managers to manipulate earnings can be beneficial to a principal in a limited commitment setting. Dutta and Gigler (2002) demonstrate that earnings management can be beneficial as it reduces the cost of eliciting truthful forecasts. Similarly, Demski, Frimor, and Sappington $(2004,2006)$ show that the principal may prefer a less accurate auditing process. Different to these studies, however, in my setting earnings management is always detrimental to the principal.

The remainder of the paper is organized as follows. Section 2 presents the basic notation and Section 3 
illustrates the optimal contract if the principal considers a report on the magnitude of earnings management. Section 4 explores the contracting usefulness of an indicator variable. Section 5 concludes.

\section{BASIC NOTATION AND MODEL STRUCTURE}

\subsection{Agent's Actions, Performance Report, and Preferences}

I consider a single-period model in which, at date $o$, a risk-neutral principal hires a risk-averse agent to provide effort at date 1 in return for compensation at date 2. The agent expends effort in two tasks, and his choice of effort levels is characterized by $(a, e)^{\prime} \in$

$\mathbb{R}^{2}$. While $a$ represents productive effort, $e$ is manipulative effort, i.e., effort that improves his performance measure but contributes nothing to the principal's gross payoff. The agent's personal cost of effort is assumed to be $\kappa(a, e)=1 / 2\left(a^{2}+\gamma^{-1} e^{2}\right)$ where $\gamma \in \mathbb{R}^{+}$parameterizes the agent's personal cost of exerting manipulative effort (i.e., the agent's cost of performing earnings management decreases with $\gamma$ ).

At date 2, a report on the agent's performance is released (e.g., firm or divisional profit). The measure is influenced by the agent's actions and random noise, i.e.,

$y=m_{a} a+m_{e} e+\varepsilon$,

where $m_{i}$ is the sensitivity of report $y$ to action $i=a, e$, and $\varepsilon \sim \mathrm{N}\left(\mathrm{o}, \sigma^{2}\right)$. The agent receives his compensation $w \in \mathbb{R}$ at date 2 , and his utility for compensation and effort is

$u(w, a, e)=-\exp [-r(w-\kappa(a, e))]$,

where $r$ is his measure of risk aversion.

At date $\mathrm{o}$, the principal offers the agent contract $z$. Without loss of generality, I assume that the agent has a net reservation wage of zero, i.e., he will not accept contract $z$ unless $\mathrm{E}[u(z, a, e)] \geq-1$.

The gross terminal value of the firm is realized at date $t \gg 2$ and represented by $x$. Because of the long delay between the agent's actions at date 1 and the realization of the terminal value, the agent is supposed to be no longer employed by the firm, such that outcome $x$ is not contractible. For simplicity, and for emphasis, the expected gross terminal value of the firm is assumed to be linear in the agent's productive effort, represented by $\mathrm{E}[x \mid a, e]=b a$, where $b \in \mathbb{R}$. Hence, the expected net terminal value is

$$
\Pi=\mathrm{E}[x-w \mid a, e] .
$$

\subsection{Auditing Technology and Auditor's Report}

At date 2, an auditor evaluates management assertions and issues a report. I consider two audits that differ in their respective objectives, the applied technology, and the collected evidence. For convenience, while an external auditor performs an audit on the financial statements of the firm, an internal auditor appraises the firm's control framework and governance processes. Moreover, whereas the external auditor releases, among other information, an estimate of the impact of earnings management on reported earnings, the internal auditor issues a report on the compliance with firm policies and procedures, which includes his (noisy) conclusion regarding the existence of earnings management.

I assume that the external auditor applies technology $g$ (comprising, e.g., audit planning, test of internal controls, substantive analytical procedures, substantive tests of detail, or roll-forward procedures $^{6}$ ) to a collected set of audit evidence $\mathbf{y}_{\mathrm{g}} \in \mathbb{R}^{n}$ (e.g., management assertions, invoices, bank statements, contracts, disclosures) to obtain a report $y_{e}$ on the magnitude of earnings management. Mathematically, $g: \mathbb{R}^{\mathrm{n}} \rightarrow \mathbb{R}$, where $g\left(\mathbf{y}_{\mathrm{g}}\right)=y_{\mathrm{e}}=m_{e} e$ $+\varepsilon_{e}, \varepsilon_{e} \sim \mathrm{N}\left(0, \sigma_{e}^{2}\right)$ is the noise inherent in $y_{e}$, and $\operatorname{Cov}\left[\varepsilon, \varepsilon_{e}\right]=\rho \sigma \sigma_{e} .{ }^{7}$

Second, the internal auditor applies technology $h$ to audit evidence $\mathbf{y}_{h} \in \mathbb{R}^{m}$ to assess the existence of earnings management, i.e., $h: \mathbb{R}^{m} \rightarrow\{0,1\}$, with $h\left(\mathbf{y}_{h}\right)=\psi$. Report $\psi$ is a noisy signal that indicates whether the agent exerted manipulative effort (i.e., $\psi$ is an indicator variable). More specifically, I assume

$\operatorname{Pr}(\psi=1 \mid e \neq 0)=\varphi$ and $\operatorname{Pr}(\psi=1 \mid e=0)=\theta$, i.e., given non-zero manipulative effort, with probability $\varphi$ the report signals that the agent performed

\footnotetext{
6 Baetge, Melcher, and Schulz (2008) offer an overview of contemporaneous auditing methods.

7 Considering performance measure $y_{\mathrm{e}}$ is without loss in generality, since one can transform any non-aligned measures $y$ and $y_{\mathrm{e}}^{\prime}=$ $m_{a} a+m_{e} e^{\prime}+\varepsilon_{e}{ }^{\prime}$ with $\varepsilon_{e}^{\prime} \sim \mathrm{N}\left(0, \sigma_{\mathrm{e}^{2}}\right)$, and $\operatorname{Cov}\left[\varepsilon, \varepsilon_{e}\right]=\rho^{\prime} \sigma \sigma_{e}^{\prime}$, into equivalent statistics $y$ and $y_{e}$.
} 
window dressing. On the other hand, even though the agent refrained from exerting manipulative effort, with probability $\theta$ the report signals window dressing. Hence, $\theta$ measures the Type I error and $1-\varphi$ the Type II error. Without loss in generality, $\varphi \geq \theta$, i.e., given $\psi=1$ the conditional probability that the agent exerted manipulative effort is larger than the conditional probability that the agent did not exert manipulative effort. ${ }^{8}$ Then, $\varphi / \theta \in[1, \infty)$, reflects the informativeness of signal $\psi$ with respect to the agent's manipulative effort. For simplicity, I assume that $\operatorname{Cov}[y, \psi]=0$, i.e., the noise associated with measuring the agent's performance is independent from the noise associated with the indicator for the agent's manipulative effort.

Several remarks are in place: First, the focus of the analysis is on the effectiveness of a report on the magnitude versus the existence of earnings management to prevent earnings manipulation. To achieve the most parsimonious model for this endeavor, I refrain from making assumptions concerning the collected evidence $\mathbf{y}_{\mathrm{g}}$ and $\mathbf{y}_{h}$, the auditing technologies $g$ and $h$, and the cost of both audits. Moreover, I neglect incentive problems regarding application of the auditing technology and revelation of the outcome of this process by the auditor, and assume that the indicator variable is insensitive with respect to the magnitude of the manipulative effort (i.e., $\psi$ is not informative with respect to $e$ if $e>0$ ). While the absence of strategic actions of the auditors can be justified by a sufficient degree of independence, the insensitivity follows from the focus on the control framework and governance processes of the internal audit. Second, the results on the effectiveness of both reports to prevent earnings management can give rise to an analysis of the combination of both audits, which is, however, beyond the scope of this paper. Finally, the uncertainty regarding the outcome of each audit report reflects the difficulty in assessing the existence and magnitude of earnings management, respectively.

\subsection{Agent's Contract}

At date $\mathrm{o}$, the principal offers a linear compensation contract to the agent. With the external auditor's report on the magnitude of earnings management, the contract is represented by $z_{e}=(f, v, \alpha)$, where $f$ is the agent's fixed wage, $v$ is the incentive rate for the

${ }^{8} \varphi \geq \theta$ does not imply that Type II error is smaller than Type I error. $\varphi=.2$ and $\theta=.1$ is an obvious example. "filtered" performance measure, and $\alpha$ is the degree to which the disclosed earnings manipulations are removed from the reported performance. Hence, the agent's compensation, given reports $y$ and $y_{e}$, and contract $z_{e}$, is

$$
w\left(y, y_{e}, Z_{e}\right)=f+v\left(y-\alpha y_{e}\right),
$$

where $y-\alpha y_{e}$ is the filtered performance measure. Here, the assumptions regarding compensation contract, preferences, and random noise are consistent with a two-task, two-performance measure LEN-model.

In a similar vein, given the internal auditor's report on the existence of earnings management, the principal offers a linear compensation contract represented by $z_{i}=(f, v, p)$ where $p$ is the agent's penalty if report $\psi$ indicates that the agent performed window dressing. Hence, the agent's compensation, given reports $y$ and $\psi$, and contract $z_{i}$, is

$$
w\left(y, \psi, z_{i}\right)=f+v y-p \psi .
$$

In this setting, the linear contract assumption rules out (i) that the principal pays a bonus to the agent if there is no signal regarding earnings management (i.e., $\psi=0$ ), and (ii) that the incentive rate $v$ for the performance report $y$ depends on $\psi$. While (i) tends to be practically irrelevant, the independence assumption (ii) is, e.g., common to the LEN setting. ${ }^{9}$

\section{IMPACT OF A REPORT ON THE MAGNITUDE OF EARNINGS MANAGEMENT}

\subsection{Standard Model}

Initially, suppose that no auditor's report is available for contracting..$^{10}$ Then, the principal's contract is represented by $z=(f, v)$ and the agent's certainty equivalent is

$$
\begin{aligned}
& \mathrm{CE}(z, a, e)=\mathrm{E}[w \mid z, a, e]-\kappa(a, e)-1 / 2 r \\
& \operatorname{Var}[w \mid z, a, e] \\
& =f+v\left(m_{a} a+m_{e} e\right)-1 / 2\left(a^{2}+\gamma^{-1} e^{2}\right)-1 / 2 r \\
& v^{2} \sigma^{2} .
\end{aligned}
$$

\footnotetext{
9 With exponential utility, normally distributed performance measures, and an infinite lower bound on net consumption the Mirrlees problem arises (Mirrlees 1974), i.e., by using a penalty contract the principal may approximate arbitrary closely first best. See Christensen and Feltham (2005), Section 19.1. In my setting, however, an effort allocation problem exists and the signal $\psi$ is a binary variable.

10 This benchmark setting is equivalent to the window-dressing in Feltham and Xie (1994).
} 
Differentiating (3) with respect to $a$ and $e$ and solving the first-order conditions establishes that the agent chooses actions

$$
a^{\dagger}=m_{a} v \text { and } e^{\dagger}=\gamma m_{e} v .
$$

Interestingly, the productive effort $a^{*}$ and the manipulative effort $e^{\dagger}$ are both driven by the incentive rate $v$. More specifically, the agent does not perform window dressing if at least one of the following three conditions is met:

(i) the performance measure is not manipulable (i.e., $m_{e}=0$ );

(ii) the cost to the agent of manipulating the performance measure is excessively high (i.e., $\gamma \rightarrow 0$ );

(iii) the principal does not offer performancecontingent pay (i.e., $v=0$ ).

Given the agent's outside opportunities, it is optimal for the principal to select a fixed wage $f^{i}$ such that $\mathrm{CE}\left(z, a^{\dagger}, e^{t}\right)=0$. Substituting (4) into (3), setting it equal to zero, solving for $f^{t}$, and substituting it and (4) into (1) yields the unconstrained objective function of the principal. Differentiating with respect to $v$ gives the following optimal incentive rate in the benchmark setting without the indicator variable. Substituting the optimal incentive rate into (1) yields the expected net payoff to the principal:

$$
\begin{aligned}
& v^{\star}=\left(m_{a}{ }^{2}+\gamma m_{e}{ }^{2}+r \sigma^{2}\right)^{-1} b m_{a}, \\
& \Pi^{+}=1 / 2\left(m_{a}{ }^{2}+\gamma m_{e}{ }^{2}+r \sigma^{2}\right)^{-1} b^{2} m_{a}{ }^{2} .
\end{aligned}
$$

Using (5a) in (4) establishes that the principal tolerates agent window dressing in the standard model. The key to this result is that precluding window dressing by setting $v=0$ results in an even lower expected net payoff to the principal.

\subsection{Contract on Filtered Performance Measure}

With the report $y_{e}$ on the magnitude of earnings management, the agent's compensation is characterized by (2a), and his certainty equivalent is

$$
\begin{aligned}
& \mathrm{CE}\left(\mathrm{z}_{e}, a, e\right)=f+v\left[m_{a} a+(1-\alpha) m_{e} e\right]-1 / 2 \\
& \left(a^{2}+\gamma^{-1} e^{2}\right)-1 / 2 r v^{2}\left(\sigma^{2}-\alpha \rho \sigma \sigma_{e}+\alpha^{2} \sigma_{e}^{2}\right) .
\end{aligned}
$$

Differentiating (6) with respect to $a$ and $e$, and solving the first-order conditions yields the agent's optimal action choices (T.1) in Table 1. Obviously, setting $\alpha=1$ removes any incentives to manipulate earnings without having detrimental effects on the productive effort choice.

The principal selects the fixed wage $f$ such that $\mathrm{CE}\left(z_{e}, a^{e}, e^{e}\right)=0$. Substituting $f^{e}$ and (T.1) into (1), differentiating with respect to $v$ and $\alpha$, and solving for the choice variables yields the optimal contract parameters as characterized by (T.2). Finally, substituting (T.2) into (T.1) yields the agent's manipulative effort (T.3), given the principal's optimal contract choice. ${ }^{11}$

\section{Table 1:}

\section{Two Action-informative Performance Measures and the Agent's Manipulative Effort:}

\begin{tabular}{l}
$a^{e}(v)=m_{a} v$ and $e^{e}\left(v, v_{e}\right)=\gamma m_{e}(1-\alpha) v$, \\
\hline$v^{e}=D^{-1}\left(\gamma m_{e} e^{2}+r \sigma_{e^{2}}\right) b m_{a} \quad$ and \\
$\alpha^{e}=\left(\gamma m_{e^{2}}+r \sigma_{e^{2}}\right)^{-1}\left(\gamma m_{e^{2}}+r \rho \sigma \sigma_{e}\right)$, with \\
\hline$D=\left(m_{a^{2}}+\gamma m_{e^{2}}+r \sigma^{2}\right)\left(\gamma m_{e^{2}}+r \sigma_{e^{2}}\right)-\left(\gamma m_{e^{2}+r}+r \sigma \sigma_{e}\right)^{2}$, \\
\hline$e^{e}=D^{-1} r \sigma_{e} \gamma m_{e}\left(\sigma_{e}-\rho \sigma\right) b m_{a}$.
\end{tabular}

Following (T.3), and as demonstrated by Feltham and Xie (1994), it is not optimal to eliminate the agent's manipulative effort unless the second measure is noiseless (i.e., $\sigma_{e}=0$ ), or for a knife-edge relation of the reports' noise (i.e., $\sigma_{e} / \sigma=\rho$ ). According to Datar, Cohen Kulp, and Lambert (2001), while the principal may choose the weight on the external report such that filtered profit (i.e., $y-\alpha y_{e}$ ) is congruent to the expected payoff (i.e., by setting $\alpha=1$ ), this contract parameter will, in general, impose too much risk on the agent. Moreover, comparing the agent's manipulative effort yields

$e^{\dagger}-e^{e}=\left(\mathrm{m}_{a}{ }^{2}+\gamma m_{e}{ }^{2}+r \sigma^{2}\right)^{-1} D^{-1}\left[m_{a}{ }^{2}+r \sigma(\sigma-\rho\right.$ $\left.\left.\sigma_{e}\right)\right]\left[\gamma m_{e}{ }^{2}+r \rho \sigma \sigma_{e}\right] \gamma m_{e} b m_{a}$,

and the sign of this difference is ambiguous. In particular, necessary conditions for a negative sign are a noisy auditor's report (i.e., $\sigma_{e} \neq 0$ ) that is correlated with the performance measure (i.e., $\rho \neq 0$ ). Hence, filtering manipulated earnings from the agent's performance measure via a report on the magnitude of earnings management can actually increase the agent's window-dressing effort. Key to this result is the noise inherent in the auditor's report, and the correlation with unfiltered performance. Hence, the weight $\alpha$ is not only chosen to improve the congru-

${ }^{11}$ Since I am only interested in the impact of the auditor's report on the agent's manipulative effort, I omit the expected net payoff to the principal in Table 1. 
ence of the overall performance measure, but also to optimize the risk imposed on the agent. Thus, with a report on the magnitude of earnings management, the principal prevents earnings manipulation only for knife-edge cases, and including this report in the agent's contract may actually increase the level of earnings management.

\section{IMPACT OF A REPORT ON THE EXISTENCE OF EARNINGS MANAGEMENT}

\subsection{Optimal Agent's Action Choices}

Including the indicator variable $\psi$ in the agent's contract yields a subtle relation between the agent's choice of manipulative effort and his expected utility. For example, given that the agent performed window dressing (i.e., $e \neq 0$ ), his compensation from $y$ is normally distributed with $\mathrm{N}\left(f+v \mathrm{E}[y \mid a, e \neq 0], v^{2} \sigma^{2}\right)$. In addition, with probability $\varphi$ the internal report is $\psi=1$, and penalty $p$ reduces the agent's compensation. On the other hand, given that the agent abstained from window dressing (i.e., $e=0$ ), his compensation from $y$ is normally distributed with $\mathrm{N}\left(f+v \mathrm{E}[y \mid a, e=0], v^{2} \sigma^{2}\right)$, and with probability $\theta$ penalty $p$ reduces his compensation. Lemma 1 characterizes the agent's certainty equivalent for the two cases where the agent performs window dressing (i.e., $\mathrm{CE}_{e}(z i, a, e)$ ), and where he abstains from window dressing (i.e., $\mathrm{CE}_{o}\left(Z_{i}, a\right)$ ). ${ }^{12}$ Lemma $1:{ }^{13}$ Given that the compensation contract induces a penalty $p$ if the indicator variable signals earnings management, the agent's certainty equivalent is characterized by

$$
\begin{aligned}
& \mathrm{CE}_{e}\left(z_{i}, a, e\right)=f+v\left(m_{a} a+m_{e} e\right)-\kappa(a, e)- \\
& 1 / 2 r v^{2} \sigma^{2}-r^{-1} \ln [1-\varphi(1-\exp [r p])],
\end{aligned}
$$

$$
\begin{aligned}
& \mathrm{CE}_{o}\left(z_{i}, a\right)=f+v m_{a} a-\kappa(a, o)-1 / 2 r v^{2} \sigma^{2}- \\
& r^{-1} \ln [1-\theta(1-\exp [r p])] .
\end{aligned}
$$

The first two terms in the agent's certainty equivalent reflect his expected compensation, the third term reflects his effort cost, the fourth term captures the risk premium for the uncertain compensation based on $y$, and the last term reflects the expected penalty plus the risk premium for the uncertain compensation based on $\psi$. The latter term is, in

12 The subscript indicates the setting with earnings management (i.e., e) and without earnings management (i.e., o).

13 The propositions are proven in the Appendix. general, negative (i.e., for $p>0$, the penalty decreases the agent's certainty equivalent), and a higher risk aversion (i.e., $r$ ), a larger penalty (i.e., $p$ ), and larger probabilities for an earnings management signal (i.e., $\varphi$ and $\theta$ ) result in a larger expected value and risk premium for the penalty. Differentiating (7a) and (7b) with respect to $a$ and $e$ and solving the first-order conditions results in the following agent's action choices:

$$
a_{e}^{*}=a_{o}^{*}=m_{a} v, e_{e}^{\ddagger}=\gamma m_{e} v, \text { and } e_{o}^{*}=0,
$$

Interestingly, for an exogenous incentive rate $v$, the agent's choice of manipulative effort has no direct impact on the productive effort in the two settings (compare (8) with (4)). The key to this result is the additive separability of the performance report $y$, the independence of the agent's effort costs, and the independence of $v$ from $\psi$. As a consequence, given that the agent performs window dressing, its amount is independent of the penalty $p$.

Given contract $z_{i}$, and the optimal action choices according to (8), the agent does not perform window dressing if $\mathrm{CE}_{e}{ }^{*}\left(z_{i}\right) \leq \mathrm{CE}_{o}^{*}\left(z_{i}\right)$. Lemma 2 summarizes these results.

Lemma 2: (a) The agent does not exert manipulative effort if the principal offers a contract $Z_{i}$ with relatively weak effort incentives, i.e., if

(9a) $v \leq v^{c}(p)=\left\{2\left(r \gamma m_{e}^{2}\right)^{-1} \ln \left(\frac{1-\varphi(1-\exp [r p])}{1-\theta(1-\exp [r p])}\right)\right\}^{1 / 2}$

(b) The principal cannot prevent the agent from choosing $e \neq 0$ via penalty contract (2b), if

$$
v>\left\{2\left(r \gamma m_{e}^{2}\right)^{-1} \ln [\varphi / \theta]\right\}^{1 / 2} .
$$

Equivalent to (9a), the agent does not exert manipulative effort for a sufficiently large penalty, i.e., if

$$
p \geq p^{c}(v)=r^{-1} \ln \left(\frac{(1-\theta) \exp \left[1 / 2 r \gamma m_{e}^{2} v^{2}\right]-(1-\varphi)}{\varphi-\theta \exp \left[1 / 2 r \gamma m_{e}^{2} v^{2}\right]}\right)
$$

Intuitively, with a sufficiently large penalty, the principal deters the agent from performing window dressing.

Observe that the critical incentive level $v^{c}(p)$ increases with the penalty $p$. Therefore, by increasing the penalty $p$, the principal is able to offer stronger effort incentives $v$ without inducing manipulative effort. However, the threshold $v^{c}(p)$ increases in $p$ at a decreasing rate, and, in the limit, following 
Lemma 2 (b), an infinitely large penalty results in a finite threshold incentive rate. Moreover, $\varphi=\theta$ yields $v^{c}(p)=o$, i.e., independent of the size of the penalty $p$, any positive incentive rate $v$ results in the agent exerting manipulative effort. Of course, the key to this result is that $\varphi=\theta$ results in an indicator variable $\psi$ that is uninformative about the agent's manipulative effort.

\subsection{Optimal Contract Parameters}

Subsequently, I consider the principal's contracting choice, given the agent's anticipated choice of productive and manipulative effort. First, I analyze the optimal contract if the penalty does not keep the agent from exerting manipulative effort. Then, I consider the optimal contract if the penalty is sufficiently large to prevent window dressing. In the first (second) setting, I initially assume that the penalty is too small (large enough) to prevent window dressing, and later confirm that the optimal incentive rate and the optimal penalty meet the conditions. In Section 4.3, I compare both settings to identify conditions under which it is beneficial to the principal to keep the agent from exerting manipulative effort.

\section{(a) Agent Exerts Manipulative Effort}

With a relatively small penalty (i.e., for $p<p^{c}$ ), the agent is motivated to exert manipulative effort. Then, the expected net payoff to the principal is

$$
\begin{aligned}
& \Pi_{e}\left(z_{i}, a, e\right)=\mathrm{E}[x \mid a, e]-\mathrm{E}[w \mid a, e]= \\
& =b a-f-v\left(m_{a} a+m_{e} e\right)+\varphi p .
\end{aligned}
$$

The agent accepts the contract if it guarantees his reservation wage (i.e., $\mathrm{CE}_{e}^{\star}\left(z_{i}\right) \geq 0$ ). The principal selects the salary $f_{e}^{*}$ such that $\mathrm{CE}_{e}{ }^{\star}\left(z_{i}\right)=0$. Substituting $f_{e}^{*}$ and (8) into (10) gives the principal's unconstrained decision problem. Differentiating it with respect to $v$ and $p$ and solving the first-order conditions results in the following optimal contract parameters. Substituting $f_{e}^{\star},(8)$, and (11a) into (1) yields the expected net payoff to the principal.

Proposition 1: Given that a relatively small penalty does not prevent the agent from window dressing, the optimal incentive rate, penalty, and the principal's expected net payoff are characterized by

(11a) $v_{e}{ }^{*}=\left(m_{a}^{2}+\gamma m_{e}{ }^{2}+r \sigma^{2}\right)^{-1} b m_{a}$,

$$
p_{e}^{*}=0 \text {, and }
$$

(11b) $\quad \Pi_{e^{*}}^{*}=1 / 2\left(m_{a}^{2}+\gamma m_{e}^{2}+r \sigma^{2}\right)^{-1} b^{2} m_{a}^{2}$.
An interesting aspect of (11a) and (11b) is that they are the same as in the setting without the indicator variable ((5a) and (5b)). More specifically, given that the penalty is not sufficiently large to prevent the agent from exerting manipulative effort the indicator variable has no value to the principal. The key is that while $\psi$ imposes incentive risk on the agent, a penalty $p<p^{c}$ does not influence the agent's effort choice (see (8)). Therefore, given that there is no benefit to the principal of using the indicator variable $\psi$, her optimal choice is to select $p_{e}^{*}=0$.

\section{(b) Principal Prevents Manipulative Effort}

I now consider the setting where the penalty is sufficiently large to keep the agent from exerting manipulative effort, i.e., $p \geq p^{c}(v)$ such that $e_{0}^{\ddagger}=0$. Then, the principal's expected net payoff is ${ }^{14}$

$$
\Pi_{o}\left(z_{i}, a\right)=b a-f-v m_{a} a+\theta p .
$$

The agent accepts the contract if it guarantees his reservation wage, i.e., if $\mathrm{CE}_{o}^{*}\left(z_{i}\right) \geq 0$. The principal selects the fixed wage $f_{o}^{\ddagger}$ such that $\mathrm{CE}_{o}^{*}\left(Z_{i}\right)=0$. Moreover, the principal chooses $p_{o}^{\ddagger}(v)=p^{c}(v)$, which imposes the least incentive risk on the agent while preventing earnings management. Substituting $f_{o}^{*}, p_{o}^{*}(v)$, and (8) into (12) gives the principal's unconstrained decision problem. Differentiating it with respect to $v$ yields the first-order condition for the optimal incentive rate as characterized by Proposition 2. Solving (13) for $v_{o}{ }^{*}$, and substituting it into $p_{o}^{*}(v)$ yields the optimum penalty $p_{o}^{*}$. Finally, substituting $v_{o}^{*}$ together with $f_{o^{*}}$ and (8) into (12) results in the expected net payoff $\Pi_{o}{ }^{*}$ to the principal.

Proposition 2: Given that a relatively large penalty prevents the agent from exerting manipulative effort, the optimal incentive rate $v_{o}^{*}$ is implicitly given by

(13) $b m_{a}-\left(m_{a}^{2}+r \sigma^{2}\right) v_{o}{ }^{\ddagger}+\theta(1-\theta) \gamma m_{e}{ }^{2} v_{o}{ }^{*}$ $\exp \left[1 / 2 r \gamma m_{e}^{2}\left(v_{o}^{*}\right)^{2}\right] \times\left\{\left((1-\theta) \exp \left[1 / 2 r \gamma m_{e}^{2}\left(v_{o}^{*}\right)^{2}\right]-\right.\right.$ $\left.(1-\varphi))^{-1}-\left(\varphi-\theta \exp \left[1 / 2 r \gamma m_{e}^{2}\left(v_{o}^{*}\right)^{2}\right]\right)^{-1}\right\}=0$.

With (13), except for some special cases, a closed form solution for $v_{0}^{*}$ is hard to find. Corollary 1 compares the incentive rate with the benchmark setting where the principal can preclude earnings

14 Observe that for $e=0$, the probability that $\psi$ signals earnings management is $\operatorname{Pr}(\psi=1 \mid e=0)=\theta$, i.e., with probability $\theta$ the penalty accrues to the principal. 
management at no cost (see Section 3.1). Corollary 1: In general, the optimal incentive rate is less than or equal to the incentive rate $v^{*}$ for the setting where the principal costlessly precludes earnings management, i.e.,

$$
v_{o}^{*} \leq v^{*} \equiv\left(m_{a}^{2}+r \sigma^{2}\right)^{-1} b m_{a}
$$

with the equality holding for $\theta=0$. In this case, the optimal penalty is given by

$$
p_{\mathrm{o}}^{\ddagger}=r^{-1} \ln \left(\frac{\exp \left[1 / 2 r \gamma m_{e}^{2}\left(v_{o}^{\ddagger}\right)^{2}\right]-(1-\varphi)}{\varphi}\right)
$$

The principal is able to preclude earnings management at no cost if, e.g., the agent cannot manipulate the performance measure (i.e., $m_{e}=0$ ), or the agent bears exceptionally large costs when exerting manipulative effort (i.e., $\gamma \rightarrow 0$ ). While the use of the indicator variable can also prevent earnings management, following Corollary 1, this outcome is achieved via a reduction of effort incentives. On the other hand, there are no negative externalities to using the indicator variable only for the special case of no Type I error (i.e., if $\theta=0$ ). Then, observing $\psi=1$ reveals with certainty that the agent exerted manipulative effort, and the penalty as given by (14b) is sufficient to keep the agent from performing earnings management.

\subsection{Contracting Usefulness of Report on the Existence of Earnings Management}

Subsequently, I compare the principal's expected net payoff when using the internal report (i.e., $\Pi_{o}^{\ddagger}$ ) with the expected net payoff when the principal refrains from using the internal report (i.e., $\Pi_{e}^{\dagger}$ ) in order to identify conditions under which contracting on the report regarding the existence of earnings management is beneficial to the principal. Due to the complexity of the optimal contract in the first setting, closed-form solutions for cutoff-values are difficult to find. To the contrary, in order to characterize the principal's tradeoff when deciding whether or not to include the internal auditor's report in the contract, I use numerical examples. In general, while I assume specific parameter values for the simulations, the implications of the subsequent results hold true for a broad set of parameter values.

\section{(a) Impact of the Indicator Variable's Meas- urement Error}

First, I analyze how the statistical properties of the indicator variable, i.e., the Type I and Type II errors ( $\theta$ and $1-\varphi$, resp.), influence the inclusion of the indicator variable in the incentive contract. It is straightforward that including $\psi$ in the agent's contract is beneficial if $\Pi_{o}^{*} \geq \Pi_{e}^{*}$. Figure 1 illustrates the expected net payoff to the principal for varying values of $\varphi \in[\theta, 1]$ in the setting with $\left(\Pi_{o}^{*}\right)$ and without $\left(\Pi_{e}^{*}\right)$ the indicator variable. Here, $b=m_{a}=m_{e}=\gamma=$ $r=\sigma=1$, and $\theta=.4 .{ }^{15}$

\section{Figure 1: Expected Net Payoff to the Princi- pal in the Settings with $\left(\Pi_{o}^{*}\right)$ and without $\left(\Pi_{e}{ }^{\ddagger}\right)$ the Indicator Variable for Varying Val- ues of $\varphi$}

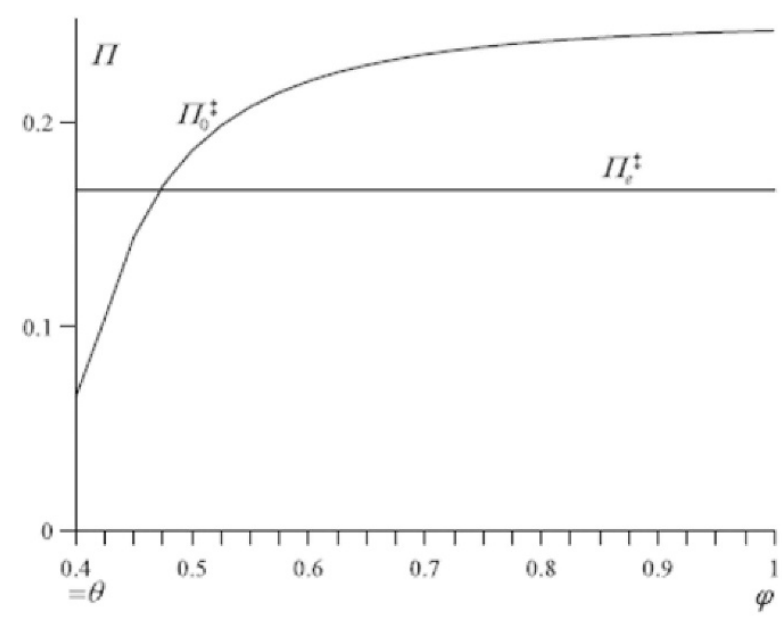

While $\Pi_{e} e^{\ddagger}$ is independent of $\varphi, \Pi_{o}{ }^{\ddagger}$ increases with a decreasing Type II error, i.e., for an increasing $\varphi$. Moreover, for $\varphi \in[.4, .4725]$ the results are such that $\Pi_{e}^{*} \geq \Pi_{o}{ }^{*}$. In particular, although $\psi$ is informative regarding $e$, the principal is better off if she did not include $\psi$ in the agent's contract. The key to this result is that for $\varphi \in[.4, .4725]$ the ratio $\varphi / \theta$ is relatively small, i.e., signal $\psi$ is only weakly informative with respect to the agent's manipulative effort, and a relatively large penalty is necessary to prevent the agent from window dressing. Because of the indicator's noisiness, a large penalty results in a large risk premium to the agent, such that the cost of preventing earnings management exceeds the benefit. On the other hand, Figure 1 illustrates that for a rela-

15 The parameter values are such that the condition in Lemma 2 (b) (i.e., $\left.v_{0}^{*} \leq\left\{2\left(r \gamma m_{e}^{2}\right)^{-1} \ln [\varphi / \theta]\right\}^{1 / 2}\right)$ is fulfilled. 
tively low Type II error (i.e., for $1-\varphi \leq .5275$ ) the principal benefits from including the indicator variable $\psi$ in the agent's contract. Hence, an internal auditor's report on the existence of earnings management will only be included in the contract and, thereby, prevent earnings management if the report is sufficiently informative with respect to the agent's manipulative effort.

\section{(b) Impact of the Performance Measure's Manipulability}

Secondly, I consider the impact of the performance measure's manipulability (i.e., $m_{e}$ ), and the impact of the agent's cost of exerting manipulative effort (i.e., $\gamma$ ) on the principal's preferences for including the indicator variable $\psi$ in the agent's contract (Figure 2). Again, $b=m_{a}=r=\sigma=1$. Moreover, $\theta=.4$ and $\varphi=.47$, i.e., I consider a setting with an only weakly informative signal $\psi$.

\section{Figure 2: Regions in the $\boldsymbol{\gamma}-\boldsymbol{m}_{e}-$ space}

Where the Principal Prefers the Use of the Indicator Variable $\left(\Pi_{o}^{*}>\Pi_{e}^{*}\right)$ and Where she Prefers not to Include the Indicator Variable in the Agent's Contract $\left(\Pi_{0}^{*}<\Pi_{e}^{*}\right)$

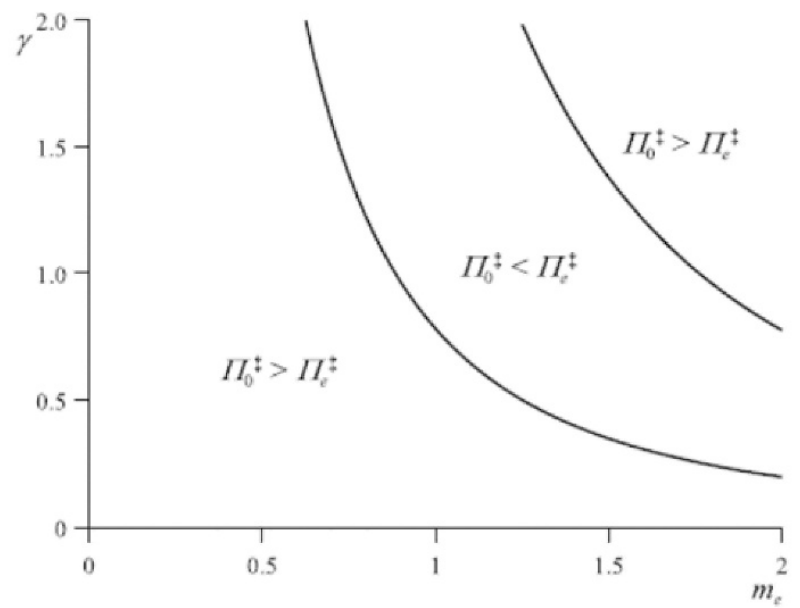

Note that the principal is indifferent with respect to including the indicator variable in the agent's contract if $m_{e}=\mathrm{o}$ or $\gamma \rightarrow \mathrm{o}$. Of course, both conditions correspond to the special cases identified in Section 3.1 where the agent does not exert manipulative effort. Following Figure 2, the principal prefers to write a contract on the indicator variable for a relatively low sensitivity $m_{e}$, or for a relatively large effort cost (i.e., for a relatively low effort cost parameter $\gamma$ ). The key to this result is that, e.g., with a low manipulability $m_{e}$, exerting manipulative effort is not attractive to the agent. Hence, a small penalty is sufficient to prevent earnings management. Also, despite the low informativeness of $\psi$, the principal prefers to bear the risk premium for this small penalty as compared to suffering from lower effort incentives and from paying the agent for his manipulative effort.

Now, for an increasing manipulability $m_{e}$, exerting manipulative effort becomes more appealing to the agent. In the setting without the indicator variable, the principal lowers the effort incentives $v_{e}^{*}$ in order to reduce earnings management. On the other hand, in the setting with the indicator variable, to prevent earnings management the principal increases the penalty $p_{o}^{\ddagger}$. Due to the low informativeness of $\psi$, and in order to limit the risk premium for the penalty $p$, the principal reduces the incentive rate $v_{0}{ }^{*}$. Ultimately, the net benefit to the principal from the effort incentives less the cost for the agent's manipulative effort exceeds the net benefit from reduced effort incentives less risk premium for the penalty. Thus, for an intermediate manipulability, the principal prefers not to contract on the indicator variable.

Finally, further increasing the report's manipulability $m_{e}$ (or, equivalently, increasing $\gamma$ ) reduces effort incentives $v_{e}^{*}$ in the setting without the indicator variable. Then, following Figure 2, the principal strictly benefits from using the indicator variable (i.e., $\Pi_{o}^{*}>\Pi_{e}^{*}$ ). The key to this result is that in the setting with the indicator variable, increasing $m_{e}$ results in lower effort incentives $v_{0}^{*}$ (i.e., $\partial v_{o}^{*} / \partial m_{e}<$ o). Then, a relatively small penalty $p_{o}^{*}$ is sufficient to prevent the agent from exerting manipulative effort (i.e., $\partial p_{o}{ }^{*} / \partial m_{e}<\mathrm{o}$ for $m_{e}$ sufficiently large). Of course, a small penalty requires a small risk premium, such that it is beneficial to the principal to use the indicator variable.

To summarize, I find that preventing earnings management by use of an indicator variable is beneficial to the principal for either a relatively low or a relatively high manipulability of the performance measure (or, equivalently, if exerting manipulative effort is either extremely costly or not costly at all to the agent). More specifically, the principal does not prevent earnings management for intermediate values of the report's manipulability.

(c) Impact of the Agent's Payoff Productivity Finally, I consider the impact of the agent's payoff 
productivity on the principal's choice of whether to include the indicator variable in the agent's contract. Corollary 2 summarizes the results.

Corollary 2: Precluding earnings management by use of the indicator variable is impossible for a relatively large payoff productivity (i.e., $\exists b$ such that $v_{o}^{\ddagger}$ $\left.>\left\{2\left(r \gamma m_{e}^{2}\right)^{-1} \ln [\varphi / \theta]\right\}^{1 / 2}\right)$.

Note that the principal imposes more incentive risk on an agent with a larger payoff productivity (i.e., the solution to (13) increases in $b$ ). As a consequence, for a relatively large payoff productivity, the solution to (13) violates Lemma 2 (b). In this case, even an infinite penalty does not deter the agent from manipulating earnings. Thus, for a relatively large payoff productivity the principal does not prevent earnings management by including the indicator variable in the contract offered to the agent.

\subsection{Impact of the Report on the Existence of Earnings Management on Effort Incentives and Penalty}

In this section, I consider the impact of contracting on the indicator variable on optimal effort incentives and penalty. Because contracting on the indicator variable deters the agent from exerting manipulative effort, one may speculate that using the indicator variable also results in stronger effort incentives as compared to the setting where the indicator variable is not used for contracting. However, Corollary 3 highlights that this is not always the case.

Corollary 3: Contracting on the indicator variable $\psi$ may result in weaker effort incentives as compared with the setting where the indicator variable is not used for contracting (i.e., there exist conditions such that $v_{o}^{*}<v_{e}^{*}$ ).

The key to Corollary 3 is that the penalty that is necessary to prevent earnings management increases with the incentive rate $v$. Hence, by decreasing the incentive rate the principal is able to lower the penalty $p_{o}{ }^{*}$, while keeping the agent from exerting manipulative effort. Ultimately, this imposes less risk of an erroneous indicator on the agent. Figure 3 illustrates Corollary 3 by revisiting the example in Section 4.3. First, following the result of Section 4.3 (a), observe that for a low informativeness of $\psi$ (i.e. for low values of $\varphi / \theta$ ), the principal prefers not to include the indicator variable in the agent's contract. More specifically, with $\theta=.4(\theta=$ .6), the principal contracts solely on $y$ if $\varphi \in[.4$, $.475](\varphi \in[.6, .675])$. Then, the effort incentive rate $v_{e}^{*}=v^{\star}$ is unaffected by the Type II error (i.e., $1-\varphi$ ), and the penalty is $p_{e^{*}}^{*}=0$.

Secondly, Figure 3 shows that under the conditions where it is optimal for the principal to use $\psi$, the incentive rate $v_{o}{ }^{\ddagger}$ increases and the penalty $p_{o}{ }^{\ddagger}$ decreases in $\varphi$. More specifically, for a smaller Type II error, the principal optimally selects a larger incentive rate and a lower penalty to motivate the agent to supply effort and to prevent him from exerting manipulative effort. Thirdly, the figure illustrates the ambiguous relation between effort incentives $v^{*}$ and the indicator's informativeness $\varphi / \theta$. In particular, a more informative measure $\psi$ yields stronger effort incentives only if $\psi$ is included in the agent's contract, i.e., following Corollary 3 , including the indicator variable in the contract may result in weaker effort incentives. Thus, preventing earnings management by the use of an indicator variable does not, in general, coincide with stronger effort incentives.

\section{Figure 3: Optimal Incentive Rate $v^{*}$ and Optimal Penalty $p^{*}$ for Varying Values of $\varphi$ and $\theta$}

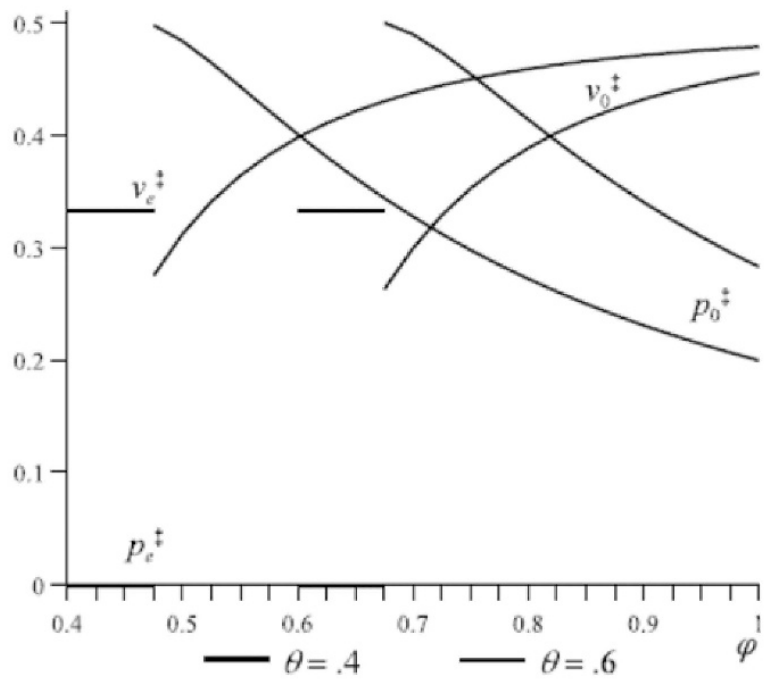

Finally, Figure 3 illustrates that increasing Type I error (i.e., $\theta$ ) broadens the conditions where it is optimal not to use the indicator variable $\psi$. Interestingly, given that the principal uses the indicator variable, a larger Type I error results in weaker effort incentives and a larger penalty. Assume, to the contrary, that the principal reduces the penalty as a consequence of a larger Type I error. A larger Type I error, however, makes it more likely that $\psi$ indicates 
earnings management, even though the agent did not exert manipulative effort. Then, given the lower penalty and the unaffected Type II error, the agent is better off by exerting manipulative effort. Thus, to the contrary, the optimal penalty increases and the incentive rate decreases with Type I error.

\subsection{Effectiveness of a Report on the Existence}

Versus the Magnitude of Earnings Management in Preventing Earnings Manipulation

The analysis in the previous sections shows that the principal can use the indicator variable $\psi$ to prevent earnings management. However, a key characteristic of this signal is that while $\psi$ is actioninformative, it is insensitive to the magnitude of the agent's manipulative effort. To understand the contracting usefulness of this indicator variable, it is helpful to compare the effectiveness of $\psi$ in preventing earnings management with the effectiveness of accounting adjustments that yield a more congruent overall performance measure, i.e., the setting with a report on the magnitude of earnings management (Section 3.2).

\section{Figure 4: Impact of the Performance Measure's Manipulability $m_{e}$ on the Magnitude of Earnings Management}

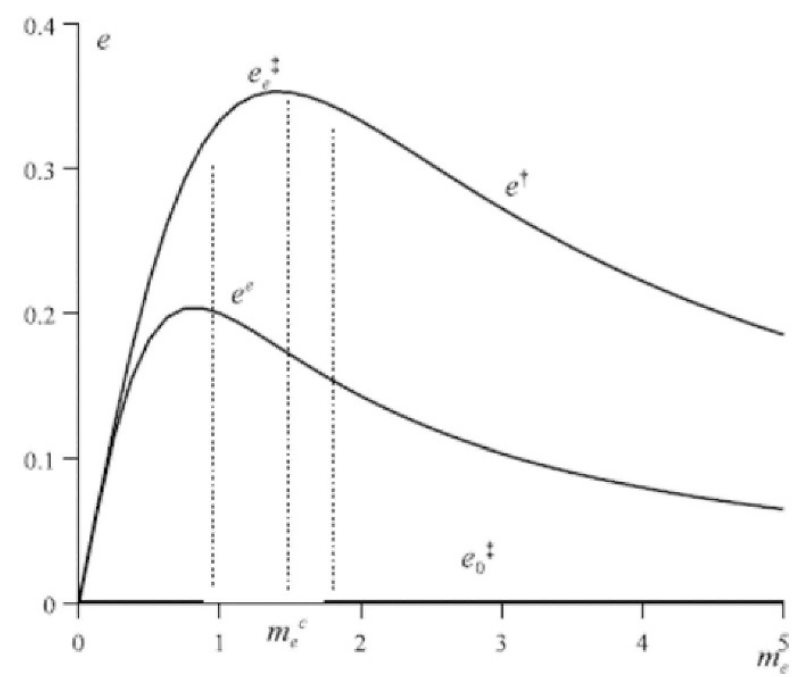

More specifically, I compare reporting system $\eta^{\Psi}$ that releases reports $y$ and $\psi$ with reporting system $\eta^{e}$ that releases reports $y$ and $y_{e}$. For the example from Section 4.3, Figure 4 illustrates the impact of the performance measure's manipulability $m_{e}$ on the magnitude of earnings management for reporting systems $\eta^{\Psi}, \eta^{e}$, and $\eta$, where the latter reports $y$.
Observe that the upper curve (with dashed and solid line) shows the agent's manipulative effort for reporting system $\eta$. For this example, reporting systems $\eta^{\Psi}$ and $\eta^{e}$ result in less earnings management as compared with $\eta$. However, while reporting system $\eta^{e}$ yields less earnings management for any manipulability of the performance measure $y$, following Section 4.3 (b), reporting system $\eta^{\Psi}$ yields less earnings management only for those conditions where the extent of manipulative effort is rather low. More specifically, under conditions of extensive earnings management, reporting system $\eta^{\psi}$ does not prevent the agent from exerting manipulative effort.

With $\eta^{\Psi}$, the agent's manipulative effort $e^{\ddagger}$ is either zero or unaffected by the indicator variable (see (8)). As indicated by Figure 4, the manipulative effort is unaffected for intermediate values of $m_{e}$, i.e., where $e^{*}$ is at its maximum. The key here is that to prevent the agent from exerting manipulative effort, the penalty $p$ must reflect the attractiveness of earnings management to the agent (i.e., $e^{i}$ is the agent's offequilibrium behavior). More specifically, to prevent extensive earnings management the principal needs to choose a relatively large penalty, which requires a large risk premium. For a weakly informative indicator variable $\psi$, however, the risk premium will be excessively large, such that the principal is better off if she does not prevent earnings management. As a consequence, a weakly informative indicator variable is only useful under conditions where the extent of earnings management is low to medium. More generally, while including a report on the magnitude of earnings management in the contract may increase the agent's manipulative effort, contracting on a report on the existence of earnings management weakly decreases the agent's manipulative effort, but the decrease will only be strict for low to medium levels of earnings management.

\section{CONCLUDING REMARKS}

In this paper, I examine the impact of an indicator variable on the agent's incentives to exert manipulative effort. I show that the principal optimally prevents earnings management if the indicator variable is sufficiently informative, the performance measure either weakly or extremely manipulative, and the agent's payoff productivity low to medium. More specifically, a noisy indicator variable is not helpful in keeping the agent from exerting manipu- 
lative effort under conditions that support excessive earnings management. In addition, I find that suppressing earnings management by use of an additional indicator variable does not naturally coincide with stronger effort incentives. Thus, even if the principal is able to prevent earnings management by threatening a severe punishment in the case of, e.g., an internal auditor's report on the existence of earnings management, occasionally, it is also optimal to reduce effort incentives.

Another way to address the earnings management problem is to focus on accounting adjustments that yield a more congruent overall performance measure. Interestingly, I find that this approach can increase the agent's effort to manipulate earnings. On the other hand, the indicator variable on the existence of earnings management weakly reduces the manager's manipulative effort, but only under conditions that result in low to medium levels of earnings management. Thus, the results suggest that under conditions that support excessive earnings management, accounting adjustments that yield a more congruent overall representation of the firm's economic value can be more effective in reducing earnings management than internal audits that provide an appraisal of whether the manager manipulated the books.

Moreover, the analysis shows that the cost to prevent earnings management increases with a manager's effort incentives, which increase in his payoff productivity. Thus, for a large sample of managers, I expect that the ones with the largest payoff productivity have the strongest effort incentives, and that they are the ones who are not refrained from exerting manipulative behavior. This implies that inferring from the observed instances of earnings management for a sub-sample of managers to the pervasiveness of earnings management in the whole economy is not possible.

Finally, considering costs for issuing the indicator variable, it is straightforward that conditions exist where the principal is better off without the additional information, especially if the report is only weakly informative regarding the existence of earnings management. Thus, a mandatory report by the external auditor on internal controls of public companies as required by Sarbanes-Oxley will be detrimental for those firms where the information is not included in the manager's compensation contract. From a management perspective, one implication of the above analysis is that under conditions of diffi- cult audits, instructing two auditors (e.g., an internal and an external auditor) to independently release reports on the compliance with firm policies regarding earnings management and on the magnitude of earnings management is most effective in precluding manipulations to the financial statements. Then, accounting adjustments based on the reported magnitude of earnings management can be used to lower the performance measure's sensitivity to window dressing, such that the compliance report is sufficiently informative to keep the manager from exerting manipulative effort.

\section{Appendix: Proof of Propositions}

\section{Lemma 1:}

Depending on the choice of manipulative effort, the agent's expected utility is given by (see also Demski, Frimor, and Sappington 2006)

(A.1a) $\quad e \neq 0:-\exp \left[-r \mathrm{CE}_{e}(\mathrm{z} ;, a, e)\right]=$ $-(1-\varphi) \exp \left[-r\left(f+v\left(m_{a} a+m_{e} e\right)-\kappa(a, e)\right.\right.$

$\left.\left.-1 / 2 r v^{2} \sigma^{2}\right)\right]-\varphi \exp \left[-r\left(f+v\left(m_{a} a+m_{e} e\right)-p\right.\right.$

$\left.\left.-\kappa(a, e)-1 / 2 r v^{2} \sigma^{2}\right)\right]$,

(A.1b) $\quad e=0:-\exp \left[-r \mathrm{CE}_{0}\left(Z_{i}, a\right)\right]=$

$-(1-\theta) \exp \left[-r\left(f+v m_{a} a-\kappa(a, 0)-1 / 2 r v^{2} \sigma^{2}\right)\right]$

$-\theta \exp \left[-r\left(f+v m_{a} a-p-\kappa(a, 0)-1 / 2 r v^{2} \sigma^{2}\right)\right]$.

Factoring out common parameters from the righthand side of (A.1a), and performing some basic mathematical transformations yields

$$
\begin{aligned}
& \text { (A.2) }-\exp \left[-r\left(f+v\left(m_{a} a+m_{e} e\right)-\kappa(a, e)\right.\right. \\
& \left.\left.-1 / 2 r v^{2} \sigma^{2}\right)\right]\{(1-\varphi)+\varphi \exp [r p]\} \\
& =-\exp \left[-r\left(f+v\left(m_{a} a+m_{e} e\right)-\kappa(a, e)-1 / 2 r v^{2} \sigma^{2}\right)\right] \\
& \exp [\ln [(1-\varphi)+\varphi \exp [r p]]] \\
& =-\exp \left[-r\left(f+v\left(m_{a} a+m_{e} e\right)-\kappa(a, e)-1 / 2 r v^{2} \sigma^{2}\right)\right] \\
& \exp \left[-r\left(-r^{-1} \ln [1-\varphi(1-\varphi \exp [r p])]\right)\right] \\
& =-\exp \left[-r\left(f+v\left(m_{a} a+m_{e} e\right)-\kappa(a, e)-1 / 2 r v^{2} \sigma^{2}\right.\right. \\
& \left.\left.-r^{-1} \ln [1-\varphi(1-\varphi \exp [r p])]\right)\right] .
\end{aligned}
$$

Replacing the right-hand side of (A.1a) with (A.2), and solving for $\mathrm{CE}_{e}\left(z_{i}, a, e\right)$ yields (7a). A similar approach results in (7b).

\section{Lemma 2:}

(a) Substituting (8) into (7a) and (7b), and simplifying, gives the certainty equivalent as

(A.3a) $\quad \mathrm{CE}_{e}^{*}\left(\mathrm{z}_{i}\right)=f+1 / 2\left(m_{a^{2}}+\gamma m_{e}{ }^{2}-r \sigma^{2}\right) v^{2}$ $-r^{-1} \ln [1-\varphi(1-\exp [r p])]$, 
(A.3b) $\quad \mathrm{CE}_{o}^{*}\left(Z_{i}\right)=f+1 / 2\left(m_{a^{2}}-r \sigma^{2}\right) v^{2}$ $-r^{-1} \ln [1-\theta(1-\exp [r p])]$.

Substituting (A.3a) and (A.3b) into $\mathrm{CE}_{e}^{*}\left(Z_{i}\right) \leq$ $\mathrm{CE}_{o}{ }^{\ddagger}\left(z_{i}\right)$, solving for $v$, and simplifying, completes the proof.

(b) From (9a), $\lim _{p \rightarrow \infty} v^{c}(p)=2\left(r \gamma m_{e}^{2}\right)^{-1} \ln [\varphi / \theta]$, i.e., a finite threshold result for an infinitely large penalty. Thus, the agent exerts manipulative effort if $v>\left(2\left(r \gamma m_{e}^{2}\right)^{-1} \ln [\varphi / \theta]\right)^{1 / 2}$.

\section{Proposition 1:}

Setting $\mathrm{CE}_{e}^{*}\left(z_{i}\right)$ according to (A.3a) equal to zero, solving for $f_{e}^{*}$, and substituting it into (10) yields

$$
\begin{aligned}
& \Pi_{e} \dot{*}(v, p)=b v m_{a}-1 / 2\left(m_{a}^{2}+\gamma m_{e}^{2}+r \sigma^{2}\right) v^{2} \\
& -r^{-1} \ln [1-\varphi(1-\exp [r p])]+\varphi p .
\end{aligned}
$$

From (A.4), the derivatives with respect to $v$ and $p$ are

(A.5a) $b m_{a}-\left(m_{a}^{2}+\gamma m_{e}^{2}+r \sigma^{2}\right) v$, and

(A.5b) $-\varphi \exp [r p](1-\varphi(1-\exp [r p]))^{-1}+\varphi$.

Using (A.5a) and (A.5b) in the first order conditions gives (11a). Substituting (11a) into (A.4) yields (11b). Finally, (11a) confirms the assumption of a weak penalty that does not prevent window dressing.

\section{Proposition 2:}

Solving $\mathrm{CE}_{o}^{*}(z)=$ o for $f_{0}^{*}$ yields

$f_{0}^{*}=-1 / 2\left(m_{a^{2}}-r \sigma^{2}\right) v^{2}+r^{-1} \ln [1-\theta(1-\exp [r p])]$.

Substituting $f_{0}{ }^{\ddagger}, p=p^{c}(v)$, and $a_{0}{ }^{*}=m_{a} v$ into (12), and simplifying, yields

(A.5) $\quad \Pi_{0}^{\ddagger}(v)=b m_{a} v-1 / 2\left(m_{a}^{2}+r \sigma^{2}\right) v^{2}-r^{-1} \ln [1$

$\left.-\theta\left(1-\exp \left[\ln \left(\frac{(1-\theta) \exp \left[1 / 2 r \gamma m_{e}^{2} v^{2}\right]-(1-\varphi)}{\varphi-\theta \exp \left[1 / 2 r \gamma m_{e}^{2} v^{2}\right]}\right)\right]\right)\right]$

$+\theta r^{-1} \ln \left(\frac{(1-\theta) \exp \left[1 / 2 r \gamma m_{e}^{2} v^{2}\right]-(1-\varphi)}{\varphi-O \exp \left[1 / 2 r \gamma m_{e}^{2} v^{2}\right]}\right)$.

$=b m_{a} v-1 / 2\left(m_{a^{2}}+r \sigma^{2}\right) v^{2}-r^{-1}\{\ln [\varphi-\theta]-\theta$

$\ln \left[(1-\theta) \exp \left[1 / 2 r \gamma m_{e}^{2} v^{2}\right]-(1-\varphi)\right]-(1-\theta) \ln [\varphi-\theta$

$\left.\left.\exp \left[1 / 2 r \gamma m_{e}^{2} v^{2}\right]\right]\right\}$.

Differentiating (A.5) with respect to $v$ gives

(A.6) $\partial \Pi_{0}^{\ddagger}(v) / \partial v=b m_{a}-\left(m_{a}{ }^{2}+r \sigma^{2}\right) v+\theta(1-\theta)$ $\gamma m_{e}{ }^{2} v \exp \left[1 / 2 r \gamma m_{e}{ }^{2} v^{2}\right] \times\left\{\left((1-\theta) \exp \left[1 / 2 r \gamma m_{e}{ }^{2} v^{2}\right]\right.\right.$

$\left.-(1-\varphi))^{-1}-\left(\varphi-\theta \exp \left[1 / 2 r \gamma m_{e}^{2} v^{2}\right]\right)^{-1}\right\}$.
Finally, $v_{0}^{*}$ is the solution to $\partial \Pi_{0}^{\ddagger}(v) / \partial v=0$.

\section{Corollary 1:}

The optimal incentive rate $v_{0} *$ is less than or equal to $v^{*}=\left(m_{a}+r \sigma^{2}\right)^{-1} b m_{a}$ if

(A.7) $\quad \theta(1-\theta) \gamma m_{e}{ }^{2} v \exp \left[1 / 2 r \gamma m_{e}^{2} v^{2}\right]$

$\times\left\{\left(\varphi-\theta \exp \left[1 / 2 r \gamma m_{e}^{2} v^{2}\right]\right)^{-1}-\left((1-\theta) \exp \left[1 / 2 r \gamma m_{e}^{2} v^{2}\right]\right.\right.$

$\left.-(1-\varphi))^{-1}\right\} \leq 0$,

where the left-hand side of (A.7) is the third addend in (A.6). (A.7) is true if

$$
\begin{aligned}
& \left(\varphi-\theta \exp \left[1 / 2 r \gamma m_{e}^{2} v^{2}\right]\right)^{-1} \\
& -\left((1-\theta) \exp \left[1 / 2 r \gamma m_{e}^{2} v^{2}\right]-(1-\varphi)\right)^{-1} \leq 0 .
\end{aligned}
$$

Simplifying (A.8), noting that (1- $\theta) \exp \left[1 / 2 r \gamma m_{e}{ }^{2} v^{2}\right]$ - $(1-\varphi)>0$ for $\varphi \neq 1$ and $\theta \neq 1$, and $\varphi-\theta \exp \left[1 / 2 r \gamma m_{e}^{2} v^{2}\right]>0$ for relatively weak effort incentives, i.e., $v<\left(2\left(r \gamma m_{e}\right)^{-1} \ln [\varphi / \theta]\right)^{1 / 2}$, results in $\exp \left[1 / 2 r \gamma m_{e}^{2} v^{2}\right] \geq 1$. Finally, substituting $\theta=0$ into (13), and solving for $v_{\mathrm{o}}^{*}$ proves the statement.

\section{Corollary 2:}

Inspecting (A.6) indicates that the right-hand side increases in $b$.

Corollary 3:

Observe that $v_{e}^{*}$ is the solution to the following first order condition:

$$
\text { (A.9) } \quad b m_{a}-\left(m_{a}^{2}+\gamma m_{e}^{2}+r \sigma^{2}\right) v=0 .
$$

Now, consider a larger incentive rate in the setting where the indicator variable is used to prevent earnings management as compared to the setting where the indicator variable is not used, i.e., $v_{0}{ }^{\ddagger} \geq v_{e}^{\ddagger}$. Comparing (13) and (A.9), a larger incentive rate follows for

$\theta(1-\theta) \gamma m_{e}{ }^{2} \exp \left[1 / 2 r \gamma m_{e}{ }^{2} v^{2}\right]\{(\varphi-\theta$ $\left.\exp \left[1 / 2 r \gamma m_{e}^{2} v^{2}\right]\right)^{-1}-\left((1-\theta) \exp \left[1 / 2 r \gamma m_{e}^{2} v^{2}\right]-\right.$ $\left.(1-\varphi))^{-1}\right\} \leq \gamma m_{e}^{2}$.

Simplifying yields

$$
\text { (A.10) } \begin{aligned}
& -\theta(1-\theta)\left((1-\theta) \exp \left[1 / 2 r \gamma m_{e}^{2} v^{2}\right]\right. \\
& -(1-\varphi))^{-1}-\exp \left[1 / 2 r \gamma m_{e}^{2} v^{2}\right]^{-1} \\
& +\theta(1-\theta)\left(\varphi-\theta \exp \left[1 / 2 r \gamma m_{e}^{2} v^{2}\right]\right)^{-1} \leq 0 .
\end{aligned}
$$

Observe that the first addend and the second addend on the left-hand side are negative since $\exp \left[1 / 2 r \gamma m_{e}^{2} v^{2}\right] \geq 1$ and $\varphi \geq \theta$. On the other hand, 
the third addend is positive if $\varphi-\theta \exp \left[1 / 2 r \gamma m_{e}{ }^{2} v^{2}\right] \geq$ o, i.e., if $1 / 2 r \gamma m_{e}^{2} v^{2} \leq \ln [\varphi / \theta]$. In this case, this addend increases in $v$. Whereas the inequality in (A.10) is true for $\exp \left[1 / 2 r \gamma m_{e}^{2} v^{2}\right]=1$, the third addend goes to plus infinity for

$\left.\lim _{\exp [1 / 2} r m e^{2} v^{2}\right] \rightarrow \varphi / \theta$.

Hence, no general statement is possible regarding the sign of the left-hand side of (A.10).

\section{Acknowledgements}

I would like to thank two anonymous referees, Rainer Niemann (editor), Thomas Pfeiffer, and Holger Daske for their helpful comments on an earlier version of the paper.

\section{References}

Arya, Anil, Jonathan Glover, and Shyam Sunder (1998): Earnings Management and the Revelation Principle, Review of Accounting Studies, 3 (1-2): 7-34.

Baetge, Jörg, Thorsten Melcher, and Roland Schulz (2008): Vermeidung von Bilanzdelikten durch (Früh-)Erkennungsmethoden - Trends in der Wirtschaftsprüfung, in: G. Herde (ed.): 3. Deggendorfer Forum zur digitalen Datenanalyse, 25-54.

Christensen, Peter O. and Gerald A. Feltham (2005): Economics of Accounting: Volume II - Performance Evaluation, Springer: New York.

Dechow, Patricia M. and Douglas J. Skinner (2000): Earnings Management: Reconciling the Views of Accounting Academics, Practitioners, and Regulators, Accounting Horizons, 14 (2): 235250.

Demski, Joel, Hans Frimor, and David Sappington (2004): Efficient Manipulation in a Repeated Setting, Journal of Accounting Research, 42 (1): 31-49.

Demski, Joel, Hans Frimor, and David E. M. Sappington (2006): Audit Error, Journal of Engineering and Technology Management, 23 (1-2): 4-17.

Datar, Srikant, Susan Cohen Kulp, and Richard A. Lambert (2001): Balancing Performance Measures, Journal of Accounting Research, 39 (1): 75-92.

Dutta, Sunil and Frank Gigler (2002): The Effect of Earnings Forecasts on Earnings Management, Journal of Accounting Research, 40 (3): 631-655.

Feltham, Gerald A. and Jim Xie (1994): Performance Measure Congruity and Diversity in Multi-task Principal/Agent Relations, Accounting Review, 69 (3) : 429-453.

Gaver, Jennifer J., Kenneth M. Gaver, and Jeffrey R. Austin (1995): Additional Evidence on Bonus Plans and Income Management, Journal of Accounting and Economics, 19 (1): 3-28.

Guidry, Flora, Andrew J. Leone, and Steve Rock (1999): Earningsbased Bonus Plans and Earnings Management by Business-unit Managers, Journal of Accounting and Economics, 26 (1-3): 113142.
Healy, Paul M. (1985): The Effect of Bonus Schemes on Accounting Decisions, Journal of Accounting and Economics, 7 (1-3): 85107.

Healy, Paul M. and James M. Whalen (1999): A Review of the Earnings Management Literature and Its Implications for Standard Setting, Accounting Horizons, 13 (4): 365-383.

Hofmann, Christian (2004): Bilanzielle Darstellungsspielräume und langfristige Anreize, Zeitschrift fir betriebswirtschaftliche Forschung, Sonderheft 51: 137-171.

Holthausen, Robert W., David F. Larcker, and Richard G. Sloan (1995): Annual Bonus Schemes and the Manipulation of Earnings, Journal of Accounting and Economics, 19 (1): 29-74.

Holmstrom, Bengt (1979): Moral Hazard and Observability, The Bell Journal of Economics, 10 (1): 74-91.

ICAEW - Institute of Chartered Accountants in England and Wales (1968): Auditors' Reports: Forms and Qualifications Statement of Auditing U1O.

IDW-Prüfungsstandard (2000): Grundsätze ordnungsmäßiger Berichterstattung bei Abschlussprïfungen (IDW PS 45o), IDWVerlag: Düsseldorf.

Ijiri, Yuji and Robert S. Kaplan (1971): A Model for Integrating Sampling Objectives in Auditing, Journal of Accounting Research, 9 (1): 73-87.

Klein, April (2002): Audit Committee, Board of Director Characteristics, and Earnings Management, Journal of Accounting and Economics, 33 (3): 375-400.

Leuz, Christian, Dhananjay Nanda, and Peter D. Wysocki (2003): Earnings Management and Investor Protection: An International Comparison, Journal of Financial Economics, 69 (3): 505-527.

Lev, Baruch (2003): Corporate Earnings: Facts and Fiction, Journal of Economic Perspectives, 17 (2): 27-50.

Merchant, Kenneth A. (1990): The Effects of Financial Controls on Data Manipulation and Management Myopia, Accounting, Organizations and Society, 15 (4): 297-313.

Mirrlees, James A. (1974): Notes on Welfare Economics, Information, and Uncertainty, in: Michael Balch, Daniel McFadden, and Shi-Yen Wu (eds.): Essays on Economic Behavior under Uncertainty, North-Holland, Amsterdam, 243-261.

Schipper, Katherine (1989): Commentary on Earnings Management, Accounting Horizons, 3 (4): 91-102.

Smith, Michael J. (2002): Gaming Nonfinancial Performance Measures, Journal of Management Accounting Research, 14: 119-133.

Watts, Ross L. and Jerold L. Zimmerman (1978): Towards a Positive Theory of the Determination of Accounting Standards, The Accounting Review, (January) 53: 112-134.

\section{Biography}

Since 2007, Christian Hofmann is chair of Managerial Accounting at the University of Mannheim. He graduated in industrial engineering and management (Technical University of Darmstadt). Hofmann received his doctoral degree in 1995 from the University of Munich and, in 2000, finished his postdoctoral lecture qualification. His areas of research include the use of accounting information in hierarchies and capital markets. 